\title{
Mutilación genital femenina: consecuencias para la salud física y mental; una violación de los derechos humanos de mujeres y niñas
}

\author{
Female Genital Mutilation: Consequences for Physical \\ and Mental Health; A Violation of the Human Rights of Women and Girls
}

Marcela del Pilar Roa Avella*

Fecha de recepción: 15 de abril del 2020

Fecha de aprobación: 15 de mayo del 2020

\section{RESUMEN}

La mutilación genital femenina (en adelante MGF) constituye una práctica aplicada a mujeres y nińas en varios lugares del mundo mediante la cual se les alteran los genitales o se remueven partes de estos, sin ninguna motivación médica. Al tener un componente cultural, de tradición, se plantea una eventual tensión con la universalidad de los derechos humanos (en adelante DDHH) para quienes afirman su relatividad. El objetivo general de este artículo es describir las consecuencias de la MGF y ponerla en contexto con el discurso de los DDHH al afirmar su universalidad.

Palabras clave: mutilación genital femenina, derechos humanos, circuncisión femenina.

\footnotetext{
Este artículo es resultado del proyecto de investigación INV DER 2556 titulado "Mutilación genital femenina, diversidad cultural y universalidad de los derechos humanos", iniciado el 25 de enero del 2018 y con fecha de terminación del 24 de enero del 2019, del Grupo de Investigación en Derecho Público, línea Derechos Humanos y Derecho Internacional Humanitario, financiado por la Vicerrectoría de Investigaciones de la Universidad Militar Nueva Granada (Bogotá, Colombia).

Citar como: Roa Avella, M. (2020). Mutilación genital femenina: consecuencias para la salud física y mental; una violación de los derechos humanos de mujeres y niñas. Via Inveniendi et Iudicandi, 15(2), 59-86. DoI: https://doi.org/10.15332/19090528/6243

** Docente investigadora de tiempo completo de la Universidad Militar Nueva Granada; magíster en Derecho Penal por la Universidad Santo Tomás, en convenio con la Universidad de Salamanca. Correo: marcela.roa @ unimilitar.edu.co mapiroa@hotmail.com. orcID: https://orcid.org/0000-0001-6307-3627?lang=es
} 


\begin{abstract}
Female genital mutilation (hereinafter FGM) is a practice applied to women and girls in various parts of the world whereby their genitals are altered or parts of them removed without any medical reason. Having a traditional cultural component, it raises a possible tension with the universality of human rights (hereinafter HHRR) for those who affirm their relativity. The overall objective of this article is to describe the consequences of FGM and place it in context with the discourse of human rights and especially the affirmation of their universality.
\end{abstract}

Keywords: female genital mutilation, human rights, female circumcision. 


\section{INTRODUCCIÓN}

La mutilación genital femenina (en adelante, MGF) implica intervenciones en los genitales femeninos que producen su alteración o remoción total o parcial, sin que de tal procedimiento se derive ningún beneficio médico. Las consecuencias y afectaciones en la salud física y mental de las mujeres sometidas a ella son variadas y con diversos niveles de intensidad, y pueden llegar incluso a afectar su salud reproductiva.

El objeto de estudio ha sido analizado en detalle por organizaciones internacionales como la ONU, Unifem, Unicef, oms y documentos que aportan valiosos elementos descriptivos en cuanto a la MGF. Por su parte, Utz-Billing y Kentenich (2008) analizan en detalle las diversas consecuencias producidas por la MGF, para revelar que estas no solo afectan la constitución física de las mujeres sometidas a ella, sino también su integridad psicológica, a la vez que produce dificultades reproductivas y obstétricas.

En materia de derechos humanos (DD. HH.), E. Katariina Paakkanen (2019) ofrece un interesante análisis en el que pone de presente que el debate no debe darse solo en términos de la MGF como una violación de los DD. HH., sino que debe trascender a las estructuras de género que subyacen en los análisis de este fenómeno.

Describiremos la práctica de la MGF, sus variantes y las diversas consecuencias derivadas de ella frente a la salud física y mental, así como los DD. HH. involucrados, con el fin de responder a la pregunta planteada: ¿existe una situación de tensión entre la MGF y la universalidad de los DD. HH? Para responder a esta pregunta, se utiliza una metodología descriptiva de una práctica social y de análisis en sus puntos de contacto con la realidad jurídica de los DD. HH.

Se inicia entonces con una descripción de la MGF, sus variantes, los riesgos que implica y las consecuencias para la salud física y mental. Luego se explican las disposiciones de las organizaciones internacionales, para referir finalmente que, de la universalidad de los DD. HH., se derivará la confirmación de la MGF como una violación de los DD. HH. de niñas y mujeres, resolviendo así la eventual tensión. 


\section{Resultados}

\section{Tipos de mutilación genital femenina}

Hemos escogido para este artículo la expresión mutilación genital femenina, ya que ha sido la recomendada por la Organización Mundial de la Salud (oms), recogida a su vez por la ONU. Sin embargo, este término no es universalmente aceptado, ya que en algunos sectores, especialmente en países donde se practica la MGF, la expresión se considera despectiva de una práctica arraigada en la cultura y la tradición.

$\mathrm{Al}$ respecto, La Barbera señala: "Por mutilación se entiende lisiar o cortar una extremidad o un órgano. Su uso implica un inequívoco juicio de valor. Desde la perspectiva occidental, estas prácticas son solo causa de enfermedades, irreparable desfiguración del cuerpo y privación permanente de la integridad física. Lo que aquí subyace es la consideración de las culturas africanas y musulmana como barbáricas, y la importancia —e incluso indispensabilidad - de Occidente para purgar dicha barbarie" (2010, p. 471). Otros autores señalan, en el mismo sentido, que el término 'mutilación' implica un juzgamiento de las culturas que lo practican y una pretensión de superioridad de la cultura occidental.

Algunos han sugerido la utilización de la expresión circuncisión femenina, al que se le ha criticado que intenta asimilar esa práctica femenina a la masculina, como quiera que en su versión masculina normalmente obedece a razones médicas (aunque es claro que también en ocasiones subyacen razones religiosas) y que, por su parte, en la versión femenina nunca hay razones médicas de por medio (Valderrama, Téllez, y Blanco, 2018):
[...] muchos autores protestan contra esa denominación de circuncisión, la cual, argumentan, sugiere erróneamente que la FGC es análoga a la circuncisión masculina. La FGC es, de hecho, bastante diferente de la circuncisión masculina en sus consecuencias físicas y sociales. (Yoder, Abderrahim, y Zhuzhuni, 2004, p. 1)

En inglés, se suma un término adicional, female genital cutting (corte genital femenino, CGF) que algunos acogen por considerarlo más neutral al no tener el sesgo violento que le critican al término 'mutilación'. En ese sentido, la Unicef indica que 
[...] la expresión "mutilación genital femenina" (MGF) consiguió un apoyo cada vez mayor a finales de los 70. La palabra 'mutilación' no solo establece una clara distinción lingüística con la circuncisión masculina, sino que también, dadas sus connotaciones claramente negativas, subraya la gravedad del acto [... En En 1991, la oms recomendó a las Naciones Unidas que adoptara esta terminología y desde entonces ha sido ampliamente utilizada en los documentos de la ONU. El uso de la palabra 'mutilación' refuerza la idea de que esta práctica es una violación de los derechos. (Unicef, 2005)

\section{Yoder, Abderrahim y Zhuzhuni precisan que}

[...] hacia la mitad de los años noventa, la oms y muchos otros grupos adoptaron la expresión mutilación genital femenina (MGF) para describir el corte de los genitales femeninos, para enfatizar el daño físico permanente hecho al cuerpo. Este término es utilizado por la mayoría de los angloparlantes, así como por muchos activistas que intervienen directamente en contra de la práctica, pero parece ser claro el cambio hacia el uso de CGF. ${ }^{1}$ (Yoder et al., 2004)

Consideramos entonces, que, si bien el término mutilación implica de suyo una expresión negativa, ello, lejos de ser una imprecisión, refleja claramente la realidad de que se trata de un fenómeno que implica violencia ejercida en contra del cuerpo de la mujer, con profundos efectos no solo físicos, sino también psicológicos, que dejan secuelas difíciles de superar.

Ahora bien, en lo que tiene que ver con las diversas modalidades de la MGF, debemos referirlas brevemente, para lo cual partimos de las caracterizaciones que han hecho de esta práctica las organizaciones internacionales.

La oms precisa que "la mutilación genital femenina (MGF) comprende todos los procedimientos consistentes en la resección parcial o total de los genitales externos femeninos, así

1 Traducción libre del original en inglés: In the mid-1990s, WHO and many other groups adopted the term female genital mutilation (FGM) to describe the cutting of female genitalia, for it emphasizes the permanent physical damage done to the body. This is the term used by the majority of English speakers, as well as many activists who direct interventions against the practice, but there seems to be a clear shift toward using FGC. 
como otras lesiones de los órganos genitales femeninos por motivos no médicos" (Organización Mundial de la Salud [OMs], 2020). Se ha clasificado la MGF en cuatro tipos (tabla 1), que responden a la modalidad de afectación que se realiza en los genitales femeninos.

Tabla 1. Tipos de mutilación genital femenina

Tipos
Tipo 1. Clitoridectomía: resección parcial o
total del clítoris (órgano pequeño, sensible y
eréctil de los genitales femeninos) y, en casos
muy infrecuentes, solo del prepucio (pliegue de
piel que rodea el clítoris). Esta modalidad en
ocasiones se refiere con la expresión 'sunna'.

Tipo 2. Escisión: resección parcial o total del clítoris y los labios menores (pliegues internos de la vulva), con o sin escisión de los labios mayores (pliegues cutáneos externos de la vulva).
En esta modalidad, la oms ha distinguido tres subtipos: tipo IIa, resección de los labios menores solamente; tipo IIb, resección parcial o total del clítoris y de los labios menores y tipo IIc, resección total o parcial del clítoris, los labios menores y los labios mayores.

Tipo 3. Infibulación: estrechamiento de la abertura vaginal que se sella procediendo a cortar y recolocar los labios menores o mayores, a veces cosiéndolos, con o sin resección del clítoris (clitoridectomía).

\begin{tabular}{ll} 
Tipo 4. Otros & Se incluyen aquí todos los demás \\
& procedimientos lesivos de los genitales externos \\
& con fines no médicos, como la perforación, la \\
& incisión, el raspado o la cauterización de la zona \\
& genital. \\
\hline
\end{tabular}

En este tipo, la subclasificación de la oms es la siguiente: tipo IIIa, resección y recolocación de los labios menores; tipo IIIb, resección y recolocación de los labios mayores.

Fuente: elaboración propia con base en la clasificación de la mutilación genital femenina de la oms; información disponible en http://www.who.int/reproductivehealth/topics/fgm/overview/es/

Es preciso mencionar también el procedimiento denominado desinfibulación, que se refiere a revertir el proceso de infibulación, y la reinfibulación, que es, a su vez, una segunda infibulación que se ejecuta cuando a una mujer se le ha practicado una desinfibulación por razones temporales, como el parto. Estos procedimientos implican 
[...] abrir el orificio vaginal cerrado de una mujer que ha sido infibulada. A menudo es necesario realizar la desinfibulación antes de las primeras relaciones sexuales, para facilitar el parto o simplemente para mejorar la salud y el bienestar de la mujer. La "reinfibulación” consiste en coser de nuevo el orificio vaginal de una mujer después de que haya sido desinfibulada (por ejemplo, después del parto), con el fin de volver a estrecharlo. También se conoce como re-sutura. (Kaplan et al., 2017, pp. 32-33)

Las diversas definiciones de la MGF enfatizan en la ausencia total de razones o justificaciones médicas en la práctica del procedimiento. La oms insiste que

[...] no tiene ningún beneficio para la salud, y daña a mujeres y niñas de muchas formas. Implica la escisión y lesión de tejido genital femenino sano y normal, e interfiere en las funciones naturales del cuerpo de las nińas y las mujeres. (Organización Mundial de la Salud [OMs], 2020)

\section{Consecuencias de la MGF}

Entre las consecuencias negativas inmediatas están el dolor, inflamación, hemorragia y fiebre; a largo plazo pueden presentarse infecciones urinarias, problemas con la menstruación, cicatrices, queloides, etc. Las consecuencias de estos procedimientos varían, teniendo en cuenta "el tipo que se practique, la pericia de quien la lleve a cabo, las condiciones higiénicas en las que se realiza, el grado de resistencia y el estado de salud general de la niña/mujer que se somete al procedimiento", sin embargo, debe concluirse que "las complicaciones pueden aparecer en todos los tipos de MGF, pero son más frecuentes en la infibulación" (UNFPA, 2019). Una práctica tan invasiva en el cuerpo necesariamente produce dolor agudo durante su ejecución y después de que ha finalizado.

Los efectos nocivos de la MGF son innegables, y en ocasiones catastróficos, ya que pueden conducir a la muerte, causada, por ejemplo, por hemorragias que no logran contenerse o infecciones que se generalizan hasta causar septicemia. 
Normalmente, se trata de una práctica no medicalizada, practicada con instrumentos no desinfectados adecuadamente, carentes de filo y a cargo de personas que, si bien son representativas en la comunidad, no tienen formación médica que les permita ejecutar el procedimiento minimizando los riesgos para la salud, lo que intensifica la posibilidad de graves consecuencias (Ballesteros, 2015).

En las condiciones en que generalmente se practica la circuncisión femenina en África, aun los tipos menos extendidos de mutilación genital pueden llevar a complicaciones potencialmente fatales, como hemorragia, infección y shock. La imposibilidad del paso de la orina debido al dolor, la hinchazón y la inflamación que siguen a la operación puede producir infección del tracto urinario. Una mujer puede sufrir de abscesos y dolor por el daño en las terminaciones nerviosas mucho después de que la herida inicial ha sanado. ${ }^{2}$

(Althaus, 1997)

Existen también consecuencias obstétricas, que van desde la mayor frecuencia en la necesidad de realización de cesáreas hasta el aumento del riesgo de muerte de la madre en el parto. Un estudio reciente ha revelado que las mujeres que se habían sometido a la MGF, en comparación con las que no lo habían hecho, se enfrentaban a un riesgo notablemente mayor de requerir una cesárea, una episiotomía y una estancia prolongada en el hospital, y también a sufrir hemorragia posparto. Las mujeres infibuladas sufren partos prolongados y obstrucción del parto, lo que a menudo se traduce en la muerte del feto y en fístulas obstétricas (UNFPA, 2019).

En particular, en la infibulación son más graves las complicaciones obstétricas, ya que impide el parto, por lo que se requiere desinfibulación previa a este: "Sin desinfibulación antes del nacimiento, puede ocurrir obstrucción del trabajo de parto que favorecen

2 Traducción libre del original en inglés: In the conditions under which female circumcision is generally performed in Africa, even the less extensive types of genital cutting can lead to potentially fatal complications, such as hemorrhage, infection and shock. The inability to pass urine because of pain, swelling and inflammation following the operation may lead to urinary tract infection. A woman may suffer from abscesses and pain from damaged nerve endings long after the initial wound has healed. 
amenazas para la vida tanto de la madre como del niño"3 (Althaus, 1997). Pero, adicionalmente, debe tenerse en cuenta que la desinfibulación afecta la disposición anatómica corriente de los genitales, su elasticidad y, en general, las características naturales requeridas para el parto en condiciones normales.

En el parto, el tejido cicatrizal de las mujeres que han sufrido la escisión puede desgarrarse. A las mujeres sometidas a la infibulación, cuyos genitales apenas disponen de abertura, es necesario practicarles un corte para permitir la salida del niño. Si no hay nadie asistiendo al parto, es posible que se produzcan desgarros perineales u obstrucción del parto. (Amnistía Internacional, 1997, p. 24)

\section{La oMs refiere que}

[...] los partos de mujeres que han sufrido MGF son significativamente más propensos a complicaciones en la cesárea, hemorragia posparto, episiotomía, hospitalización materna prolongada, resucitación del recién nacido y muerte perinatal hospitalaria que los partos de mujeres que no han sufrido MGF. ${ }^{4}$ (Organización Mundial de la Salud [oмs], 2006, p. 6)

Si bien algunos estudios señalan la dificultad de encontrar las razones por las que la MGF está relacionada con complicaciones obstétricas, se refieren, al parecer, en líneas generales, a la formación de tejido cicatrizal en zonas con alto componente vascular y nervioso. En ese sentido, Hakim ha explicado que

[...] el perineo es delicado en su estructura anatómica y es altamente vascularizado y rico en innervación. La mutilación genital es un daño irreversible con sacrificio y pérdida de la elasticidad de los tejidos afectados.

3 Traducción libre del original en inglés: Without deinfibulation before childbirth, obstructed labor may occur, causing life-threatening complications for both mother and infant.]

4 Traducción libre del original en inglés: These results show that deliveries to women who have undergone FGM are significantly more likely to be complicated by caesarean section, postpartum haemorrhage, episiotomy, extended maternal hospital stay, resuscitation of the infant, and inpatient perinatal death, than deliveries to women who have not had FGM. 
La desfiguración de los genitales externos queda evidentemente propensa a mayor incidencia de desgarres/laceraciones durante el parto y se anticipa que pueda interferir en el progreso del parto. De manera intuitiva, resulta evidente que ello contribuye a una cantidad de complicaciones obstétricas durante el parto. $^{5}$ (Hakin, 2001)

Si bien cada tipo de MGF tiene variadas consecuencias en la salud de diferente gravedad e intensidad, al tratarse de la infibulación (el caso más extremo de ella), sus efectos igualmente son más intensos. Adicionalmente, en materia de salud sexual y reproductiva, las consecuencias también están relacionadas con la afectación de los genitales, a través de procedimientos que modifican su anatomía natural y que generan cicatrices, en ocasiones queloides o infecciones, lo que puede producir también consecuencias en la salud sexual y reproductiva. Uno de los temas más polémicos ha sido el de la afectación de la posibilidad de obtener placer sexual. Las modificaciones o la pérdida de la capacidad de sentir deseo sexual o de obtener placer sexual están relacionadas no solo con las consecuencias físicas, sino también con las afectaciones psicológicas.

Gran parte de la investigación cualitativa sobre los efectos de la MGF sobre la sexualidad de la mujer ha sugerido que todos los tipos de MGF inhiben en las mujeres la satisfacción sexual y el placer. El daño físico resultante de la mutilación genital combinada con el trauma psicológico y el dolor pueden afectar la vida sexual de una mujer adulta. ${ }^{6}$ (Bennett, n. d.)

Puede afirmarse que el dolor en los genitales, derivado en ocasiones del daño a los múltiples nervios presentes en la zona, a las infecciones o cicatrices pueden implicar no solo una dificultad anatómica para mantener relaciones sexuales, sino también convertir el acto

5 Traducción libre del original en inglés: The perineum is delicate in its anatomical structure and is highly vascular and rich in nerve supply. Genital mutilation is an irreversible damage with scarification and loss of elasticity of the involved tissues. The disfigured external genitalia is obviously subjected to a higher incidence of tears/lacerations during parturition and is anticipated to interfere with the progress of labour. Intuitively, it is thus obvious that it does contribute to a number of obstetrical complications during delivery.

6 Traducción libre del original en inglés: Much of the qualitative research regarding the effects of FGM on the sexuality of women has suggested that all types of FGM inhibit sexual fulfillment and pleasure for women. The physical damage resulting from FGM combined with the psychological trauma and pain can compromise an adult woman's sexual life. 
sexual en una experiencia en extremo dolorosa. Incluso, los efectos psicológicos pueden conducir a que la mujer recree la experiencia traumática de la MGF en cualquier evento de contacto externo con sus genitales.

Los problemas sexuales como la pérdida del deseo sexual causado por la dispareunia y la capacidad reducida o nula de tener un orgasmo son frecuentes. La incontinencia u otros problemas de salud dificultan la participación en la vida social normal. (Utz-Billing y Kentenich, 2008)

Sin embargo, los estudios disponibles no parecen concluyentes. Elizabeth Heger Boyer hace un recuento de algunos y señala, entre otros, que algunos estudios indican que la MGF no implica necesariamente la pérdida del placer sexual, en especial, la posibilidad de experimentar orgasmos, a la vez que refiere otro estudio que indicó que más del $80 \%$ de las mujeres infibuladas nunca habían experimentado un orgasmo, mientras que, de las sometidas a clitoridectomía, solo el 10 \% indicó lo mismo (Boyle, 2005, p. 35).

Un estudio realizado en Burkina Faso llega a tres conclusiones importantes, relacionadas con las consecuencias físicas de la MGF: 1) todos los tipos de cortes realizados en la MGF están relacionados con cicatrización (queloide, engrosamiento de la vagina), debido al tejido sensible de los genitales; 2) se presenta relación entre el tipo de corte y las complicaciones a largo plazo, esto es, entre más severo el corte, mayor aquella probabilidad y 3) mujeres que han sufrido MGF son más propensas a infecciones del tracto reproductivo (Jones, Diop, Askew, y Kaboré, 2003, p. 227).

Deben referirse también los efectos psicológicos que se derivan de esta práctica para mujeres y nińas. Las particularidades que rodean dicha práctica de la MGF permiten imaginar los impactos psicológicos que causan. Su práctica es dolorosa per se, ya que no es común el uso de ningún tipo de anestésico y los instrumentos utilizados son rudimentarios. Algunos expertos sugieren que la conmoción y el trauma causados por la operación pueden contribuir a perfilar un comportamiento descrito como tranquilo y "dócil", que se considera positivo en las sociedades que practican la mutilación genital femenina (Amnistía Internacional, 1997). 
En muchos lugares del mundo, esta práctica se produce en niñas, por lo que, en estos casos, las consecuencias son más intensas al tratarse de un menor de edad, quien, además, sufre la mutilación a manos de un adulto, lo que produciría sentimientos de inseguridad, de abandono.

[...] generalmente las niñas están conscientes cuando se les practica la operación y para muchas es una experiencia impactante marcada por dolor agudo, miedo y confusión. La experiencia de la MGF se ha relacionado con un rango de trastornos psicológicos y psicosomáticos que incluye cambios en los hábitos alimenticios y del sueño, pérdida del apetito, pérdida o aumento excesivo de peso, ataques de pánico, dificultades en concentración y aprendizaje y síntomas de desorden de estrés postraumático.7 (Bennett, n. d., p. 137)

Algunos autores se han referido al síndrome genitally focused anxiety-depression, que "se caracteriza por experimentar una constante preocupación sobre el estado de los genitales y pánico a la infertilidad [...]” (Gomez-Limón, 2011, p. 185). No es difícil entender que la experiencia traumática de cualquier tipo de MGF dejará en la niña o mujer profundas huellas psicológicas.

\section{Gómez-Limón señala que}

[...] la pérdida de sangre, el dolor y el miedo experimentados por la mujer o la nińa cuando es mutilada [...] también puede provocar posteriormente desórdenes psicológicos y psicosomáticos, tales como angustia, alteraciones del sueño, de los hábitos alimentarios o del humor. (Gomez-Limón, 2011, p. 182)

Lo traumático de la experiencia de la MGF es innegable y, en consecuencia, podrían producirse los efectos que normalmente acompañan las experiencias traumáticas; "La mutilación genital femenina es probable que cause variados trastornos emocionales, forjando el

$7 \quad$ Traducción libre del inglés: Girls are generally conscious when the operation is performed and for many it is a shocking experience marked by acute pain, fear, and confusion. The experience of FGM has been related to a range of psychological and psychosomatic disturbances, including changes in eating and sleeping habits, loss of appetite, weight loss or excessive weight gain, panic attacks, difficulties in concentrating and learning, and symptoms of post-traumatic stress disorder. 
camino hacia desórdenes psiquiátricos, especialmente trastorno de estrés postraumático ${ }^{8}$ (Behrendt y Moritz, 2005, p. 1001).

La MGF produce impactos en la propia percepción de la niña o mujer y en cómo ella espera ser vista por el resto de su comunidad. Afectaría adicionalmente sus relaciones con los miembros de la comunidad encargados del procedimiento, que normalmente son aquellos que representan autoridad o sabiduría y que, luego de la experiencia traumática, podrían hacer que la niña pierda su confianza en quienes se supone podría depositarla.

[...] podría esperarse que el corte genital afecte la forma en que las niñas y las mujeres se entienden y perciben dentro de su sociedad, así como en su sexualidad, su confianza en los cuidadores, su autoestima y la interacción con sus parejas sexuales. ' (Jones et al., 2003, p. 220)

Es claro que los pocos estudios relacionados con los efectos psiquiátricos y psicológicos de la MGF sostienen que tal práctica produce en las mujeres diversos efectos y sentimientos que afectan su normalidad emocional, y listan una variedad de consecuencias que se resumen en la ausencia de una normalidad emocional en las mujeres sometidas a tal práctica, afectaciones en su autopercepción y trastornos psicológicos y emocionales. Poca investigación científica se encuentra disponible respecto de las consecuencias sexuales y psicológicas de la práctica. Esto se debe parcialmente a las dificultades para medir la angustia psicológica y a la aversión de las mujeres a discutir estos problemas ${ }^{10}$ (Bennett, n.d., p. 137).

8 Traducción libre del original en inglés: [...] female genital mutilation is likely to cause various emotional disturbances, forging the way to psychiatric disorders, especially PTSD.

9 Traducción libre del original en inglés: [...] genital cutting could be expected to affect how girls and women understand and perceive themselves within their society, and to affect their sexuality, their trust in caretakers, their self-esteem, and their interactions with sexual partners.

10 Traducción libre del original en inglés: little scientific research is available on the sexual and psychosocial consequences of the practice. This is partly due to difficulties in measuring psychological distress and partly due to women's reluctance to discuss these issues. 
Utz-Billing y Kentenich señalan, entre otros, que

[...] las consecuencias sociales y mentales de las mujeres después de la MGF incluyen los sentimientos de incompletud, miedo, inferioridad y supresión que influyen en la vida de las mujeres. Reportan irritabilidad crónica y pesadillas. Después de la MGF, las mujeres también presentan mayor riesgo de enfermedades psiquiátricas como depresión, psicosis, neurosis y enfermedades psicosomáticas. ${ }^{11}$ (Utz-Billing y Kentenich, 2008)

$\mathrm{Al}$ respecto, llama particularmente la atención la referencia al "sentimiento de incompletud", que define con claridad los efectos que la MGF produce en la mente de la mujer a la que se le arrebata una parte de su cuerpo, lo que precisamente desata la pérdida del sentido de individuo completo, de integridad física, que se ve alterada por la mutilación.

Los efectos psicológicos pueden incluso causar afectaciones futuras en el comportamiento; la MGF puede dejar una marca duradera en la vida y la mente de la mujer que la ha sufrido. Las complicaciones psicológicas pueden quedar inmersas profundamente en la mente subconsciente del niño y desencadenar la aparición de trastornos del comportamiento $^{12}$ (Dorkenoo, 1996).

Un estudio de Glover, Liebling, Barrett y Goodman (2017) refiere que

[...] la MGF produce un impacto de sobre la identidad de la participante como mujer; asimismo, las sobrevivientes reportaron que el procedimiento las hizo sentir menos femeninas. Esto quedó drásticamente relacionado con la idea,

11 Traducción libre del original en inglés: Mental and social consequences of women after FGM include the feelings of incompleteness, fear, inferiority and suppression that influence the whole life of women. They report chronic irritability and nightmares. Women after FGM also have a higher risk for psychiatric diseases like depressions, psychosis, neurosis and psychosomatic diseases.

12 Traducción libre del original en inglés: FGM may leave a lasting mark on the life and mind of the woman who has undergone it. The psychological complications may be sub merged deeply in the child's subconscious mind and may trigger the onset of behavioral disturbances. 
entre las mujeres, de que se les había practicado la MGF a causa de su género, de lo que se desprenden sentimientos conflictivos hacia su identidad femenina. ${ }^{13}$

Puede concluirse que la MGF no tiene ninguna justificación ni beneficio de tipo médico y que, por el contrario, produce consecuencias negativas para la salud e integridad física y mental de quienes la sufren. A esto debe sumarse que muchas mujeres no pueden expresar sus sentimientos en torno a la MGF, ya que, al tratarse de una práctica tan arraigada en la cultura de los grupos humanos que la practican, es percibida por la comunidad no solo como un evento normal sino deseable. "En muchos casos, mujeres y niñas que han sido traumatizadas por la MGF permanecen en silencio frente a su experiencia. En algunas culturas ellas no cuentan con medios socialmente aceptables para expresar sus sentimientos y angustia"14 (Bennett, n.d., p. 137).

Así las cosas, a la angustia, el terror y el dolor causados por la MGF, se suma la imposibilidad de expresar todos esos sentimientos por temor al reproche social o a la incomprensión de tales sentimientos e, incluso, a las nocivas consecuencias relacionadas con el estatus de la mujer en el seno de la colectividad, ya que esta práctica en muchas ocasiones se traduce en un ritual de tránsito a la adultez o que abre las posibilidades de contraer matrimonio; en todos esos casos, la aceptación sumisa de la práctica garantiza a la mujer una adecuada aceptación dentro el grupo.

Donde la mutilación genital femenina es ampliamente practicada, es apoyada por hombres y mujeres, por lo general sin cuestionar, y cualquiera que se aleje de la norma puede enfrentar la condena, el acoso y hasta el ostracismo. La mutilación genital femenina representa una convención social gobernada por recompensas y castigos que conforman una fuerza poderosa para perpetuar la práctica misma (Organización Mundial de la Salud [oms], 2008, p. 5).

13 Traducción libe del original en inglés: FGM impacted upon participant's identity as a woman, with survivors reporting that the procedure made them feel less feminine. This was strongly related to women's conceptualisation of FGM as being performed due to their gender, thus generating conflicted feelings towards their female identity.

14 Traducción libre del original en inglés: In many cases, women and girls who have been traumatized by FGM remain silent about their experience. In some cultures they have no socially acceptable means of expressing their feelings or distress. 
Las consecuencias psicológicas de estas prácticas no solo afectan a aquellas mujeres que han afrontado algún tipo de MGF. El miedo al rechazo social de las que no se han sometido a ella puede producir un trastorno de ansiedad social profunda y otras repercusiones negativas. Por ello, no es raro que algunas de las mujeres a las que no se les ha practicado recurran a la MGF en busca de salir de situaciones de exclusión social (Asociación Mujeres entre Mundos, 2016, p. 26).

\section{La mutilación genital femenina como violación de los derechos humanos: el contenido del derecho a la salud}

Hemos descrito las diversas consecuencias negativas que la MGF; es necesario ahora reafirmar cómo ello implica una violación del derecho a la salud y a una vida libre de violencias. Elizabeth Heger Boyle resalta en la relación entre DD.HH. y MGF un primer momento, en los años 60, específicamente cuando el Consejo Económico y Social de las Naciones Unidas solicitó a la OMS estudiar el fenómeno de la MGF, y esta organización se negó a hacerlo, argumentando que aquel tenía una naturaleza social y cultural más que médica. Este escenario cambiaría en la década de los 90, cuando la comunidad internacional se comprometió con la erradicación de la MGF (Boyle, 2005, p. 41).

Otro de los problemas planteados en la relación entre MGF y DD. HH. está dado por el concepto de soberanía, en tanto la propia Carta de las Naciones Unidas, en su preámbulo, reconoce que los asuntos sociales y culturales, incluidas las relaciones de género, son asuntos de alcance nacional sometidos a las jurisdicciones de cada país (Boyle, 2005, p. 44). A lo anterior se sumaba el arraigo que, en tradiciones y cultura, ha tenido la MGF. Esta práctica ha pasado de generación en generación en los lugares donde se produce, lo que la enmascaraba como un verdadero asunto local en el que no tendrían cabida las intervenciones de la comunidad internacional a través de sus organizaciones. Para Amnistía Internacional,

[...] varios factores han impedido que, durante muchos años, esta práctica fuera considerada como una cuestión de derechos humanos. La mutilación genital femenina es promovida por los padres y los miembros de la familia, que creen que tendrá efectos benéficos para la nińa en su vida posterior 
[...] Otro obstáculo era el hecho de que la mutilación genital femenina está arraigada en las tradiciones culturales. La intervención externa en nombre de los derechos humanos universales corría el riesgo de ser percibida como un acto de imperialismo cultural. (Amnistía Internacional, 1997, p. 37)

Se trata entonces de una práctica que durante mucho tiempo estuvo protegida por la intervención de los organismos internacionales, ya que la protege su ámbito relativo a los asuntos privados, legitimado además, en gran medida, por la tradición, el arraigo y la cultura.

Los 60 y 70 estuvieron marcados por una sensibilización cada vez mayor sobre los derechos de las mujeres en muchos lugares del mundo y las organizaciones de mujeres empezaron a liderar campańas para suscitar una mayor concienciación sobre los efectos perjudiciales de la MGF para la salud de las nińas y las mujeres. (Unicef, 2005, p. 26)

Fueron los movimientos feministas los primeros en llamar la atención acerca de la MGF, sin que ello se diera de manera pacífica porque, incluso, muchas mujeres africanas se oponían al tratamiento de la MGF como una práctica violenta, lesiva de los DD. HH. Boyle (2005) refiere cómo en la Conferencia Mundial de la Mujer celebrada en Copenhage en 1980 se produjeron protestas de mujeres africanas que tacharon las sesiones de etnocentristas.

A pesar de esa oposición, las organizaciones internacionales acogen el discurso de eliminación de la MGF, en acatamiento de la protección del derecho humano a la salud: "Los argumentos médicos fueron, entonces, la base de la intervención internacional, en contra del CGF por organizaciones como la OMS, Unicef y UNFPA" (Boyle, 2005, p. 50).

Claramente, la Convención contra todas las Formas de Discriminación en contra de las Mujeres (CEDAw, según su sigla en inglés), de 1979, supuso un punto de quiebre en el discurso de los derechos humanos de las mujeres, y ello se ha visto reforzado por las siguientes conferencias mundiales de la mujer. En ese escenario, ya desde los 80 se ha afirmado que la MGF constituye una violación de los DD. HH. de las mujeres, en tanto atenta contra su derecho a la salud y a una vida libre de violencia y discriminación, y sería, incluso, violatoria de la prohibición de la tortura. 
En cuanto a la regulación actual, podemos indicar que en los diferentes sistemas de protección de DD. HH. se reconocen los derechos a la vida y a la integridad física, psíquica y sexual, entre otros, y se rechazan todas las prácticas relacionadas con la opresión y la discriminación de las mujeres por razones de género. En el mismo sentido, las regulaciones parecen suscitar especial interés por el compromiso de los Estados, mediante la formulación e implementación de programas y planes en materia educativa que permitan luchar, en todos los niveles de formación, por eliminar patrones de discriminación y de estereotipos de género y desigualdad entre hombres y mujeres, así como las prácticas tradicionales que confirmen tal desigualdad o discriminación.

En la declaración interagencias celebrada en el 2008, la ACNUDH, UNAIDS, UNDP, UNECA, Unesco, UNFPA, UNHCR, Unicef, UNIFEM Y LA OMS afirmaron que "la mutilación genital femenina de cualquier tipo ha sido reconocida como una práctica perjudicial y una violación de los derechos humanos de las niñas y mujeres"15 (Organización Mundial de la Salud [oms], 2008, p. 8).

En la regulación actual, en el Sistema de la ONU, se destacan la Declaración Universal de los Derechos Humanos, en especial los artículos 3 (derecho a la vida) y 5 (prohibición de la tortura); el Pacto Internacional de Derechos Civiles y Políticos (Comité de Derechos Humanos, 1966), en especial los artículos 6 (derecho a la vida) y 7 (prohibición de la tortura) y la Convención sobre la Eliminación de todas las Formas de Discriminación contra la Mujer (Comité para la Eliminación de la Discriminación de la Mujer), artículo 11, literal F (Derecho a la Protección de la Salud). Adicionalmente, se resaltan la salvaguarda de la función de reproducción, la Convención contra la Tortura y otros Tratos o Penas Crueles, Inhumanos o Degradantes (Comité contra la tortura, 1984), artículo 1, en especial su definición de la tortura como todo acto por el cual se infrinja intencionadamente a una persona dolor o sufrimiento grave, ya sean físicos o mentales; la Convención sobre los Derechos del Niño (Comité de los Derechos del Niño, 1989), artículo 6, que reitera el derecho intrínseco a la vida, y el 24, que refiere el disfrute del más alto nivel posible de salud; y, finalmente, la Recomendación general núm. 14 sobre circuncisión femenina (Alto Comisionado de las Naciones Unidas para los Derechos Humanos, el Comité para la Eliminación de la Dis-

15 Traducción libre del original en inglés: Female genital mutilation of any type has been recognized as a harmful practice and a violation of the human rights of girls and women. 
criminación de la Mujer, 1990), en especial, las recomendaciones a los Estados partes para adoptar medidas para erradicar la circuncisión femenina.

Por último, la Recomendación general núm. 19 sobre la violencia contra la mujer, Alto Comisionado de las Naciones Unidas para los Derechos Humanos (1992), se refiere a las políticas que deben desarrollarse para erradicar las actitudes tradicionales según las cuales se considera a la mujer como subordinada o se le atribuyen funciones estereotipadas que perpetúan la difusión de prácticas que entrañan violencia o coacción, como la circuncisión femenina. Se señala también que en algunos Estados existen prácticas perpetuadas por la cultura y la tradición que son perjudiciales para la salud de mujeres y niños, entre las cuales se incluye la circuncisión femenina o mutilación genital. La Declaración sobre la Eliminación de la Violencia contra la Mujer (1993) señala que la MGF forma parte de las manifestaciones consideradas constitutivas de violencia contra la mujer, a la par que exhorta a los Estados a condenar la violencia contra la mujer y no invocar ninguna costumbre, tradición o consideración religiosa para eludir su obligación de procurar eliminarla.

A su vez, en el sistema interamericano existen varios instrumentos que contienen previsiones relacionadas con la protección de derechos que se ven violentados con la práctica de la MGF, a saber: la Declaración Americana de los Derechos y Deberes del Hombre (1948) se refiere, en el artículo 1, al derecho a la vida; la Convención Americana sobre Derechos Humanos (Pacto de San José de Costa Rica, 1969) se refiere en el artículo 4 al derecho a la vida y en el artículo 5, al derecho a la integridad personal, en el que además refiere la prohibición de la tortura, penas o tratos crueles, inhumanos o degradantes; el Protocolo Adicional a la Convención Americana sobre Derechos Humanos en materia de derechos económicos, sociales y culturales (Protocolo de San Salvador, 1988), en su artículo 10 establece el derecho a la salud; la Convención Interamericana para Prevenir, Sancionar y Erradicar la Violencia contra la Mujer (Convención de Belem do Pará, 1994), en el artículo 1 se refiere al concepto de violencia contra la mujer, en el 4, el derecho a que se respete la vida y la integridad física, psíquica y moral, además del derecho a no ser sometida a torturas; por su parte, el artículo 6 precisa que el derecho de toda mujer a una vida libre de violencia incluye, entre otros, "el derecho de la mujer a ser valorada y educada libre de patrones estereotipados de comportamiento y prácticas sociales y culturales basadas en conceptos de inferioridad o subordinación”; finalmente, el artículo 
8 establece como obligación de los Estados partes la adopción de medidas específicas y programas para contrarrestar prejuicios y costumbres y todo otro tipo de prácticas que se basen en la premisa de la inferioridad o superioridad de cualquiera de los géneros o en los papeles estereotipados del hombre y la mujer que legitiman o exacerban la violencia contra la mujer.

Por su parte, en el Sistema Europeo se destaca la Convención Europea de Derechos Humanos (Convención para la protección de los derechos humanos y las libertades fundamentales, 1950); se referencia en el artículo 2 el derecho a la vida y en 3, la prohibición de la tortura; el Convenio del Consejo de Europa sobre Prevención y Lucha contra la Violencia contra la Mujer y la Violencia doméstica, Convenio de Estambul, 2011), incorpora el artículo 38, referido especialmente a la MGF y en el que insta a las partes a tipificarla como delito.

Finalmente, en el sistema africano se destaca la Carta Africana de Derechos Humanos y de los Pueblos (1981), que en el artículo 4 consagra los derechos a la vida y la integridad personal, mientras que, en el 18, numeral 3, indica que el Estado se responsabiliza de eliminar la discriminación contra la mujer.

En los diferentes sistemas de protección de DD. HH. se reconocen, entre otros, los derechos a la vida y a la integridad física, psíquica y sexual y se rechazan todas las prácticas relacionadas y o derivadas de la opresión y discriminación de las mujeres, por razones de género. En el mismo sentido, las regulaciones parecen suscitar especial interés en el compromiso de los Estados, con la formulación e implementación de programas y planes en materia educativa que permitan que, en todos los niveles de formación, se luche por eliminar patrones de discriminación y estereotipos de género y de desigualdad entre hombres y mujeres.

Los principales organismos internacionales relacionados con DD. HH. han advertido que

[...] la mutilación genital femenina viola una serie de derechos humanos, principios, normas y estándares establecidos, incluidos los principios de igualdad y no discriminación basados en sexo, el derecho a la vida cuando el procedimiento termina en la muerte y el derecho a una vida libre de tortura, 
penas o tratos crueles, inhumanos o degradantes. ${ }^{16}$ (Organización Mundial de la Salud [oмs], 2008, p. 9)

En cuanto a la prohibición de discriminación por razones de pertenencia a un género, la MGF es una expresión de sociedades patriarcales y priva a las mujeres y niñas de la posibilidad de disfrutar una vida libre de violencia. Las justificaciones o legitimaciones de la MGF (pureza, higiene, preparación para el matrimonio, entre otras) son manifestaciones de una sociedad que concibe a las mujeres por debajo de los hombres y las convierte en repositorios necesarios para la procreación.

La mutilación genital femenina ha sido reconocida como discriminación basada en el sexo porque está enraizada en desigualdades y desequilibrios de poder entre hombres y mujeres, además de que inhibe a las mujeres del disfrute completo e igualitario de sus derechos humanos. Constituye una forma de violencia contra las niñas y mujeres, con consecuencias físicas y psicológicas. ${ }^{17}$ (Organización Mundial de la Salud [oms], 2008, p. 10)

La MGF es una práctica en la que se quita a las mujeres y niñas la posibilidad de decidir sobre su propio cuerpo; se les quita una parte de su corporalidad y se las modifica anatómicamente, sin que puedan participar en esa decisión, a la cual están veladamente forzadas, ya que, en ausencia de ella, no podrán ser legítimamente reconocidas como parte del grupo ni serán aptas para el matrimonio. La ausencia de la MGF convierte a la mujer en una paria dentro de su propio grupo social. "La mutilación genital femenina priva a las niñas y mujeres de tomar una decisión independiente acerca de una intervención que

16 Traducción libre del original en inglés; Female genital mutilation violates a series of well established human rights principles, norms and standards, including the principles of equality and non-discrimination on the basis of sex, the right to life when the procedure results in death, and the right to freedom from torture or cruel, inhuman or degrading treatment or punishment.

17 Traducción libre del original en inglés: Female genital mutilation has been recognized as discrimination based on sex because it is rooted in gender inequalities and power imbalances between men and women and inhibits women's full and equal enjoyment of their human rights. It is a form of violence against girls and women, with physical and psychological consequences. 
dejará efectos a largo plazo en sus cuerpos e infringe su autonomía y control sobre sus propias vidas"18 (Organización Mundial de la Salud [oms)], 2008, p. 10).

Debe añadirse que como la MGF es una práctica que se aplica en muchas ocasiones a niñas, es mayor la necesidad de sobreponerle a ella y a su arraigo en la cultura la protección sus DD. HH.; por ello, no puede perderse de vista que son las menores las principales víctimas y que es universalmente aceptado que esta práctica atenta contra las nińas y les violenta sus DD. HH. Ello las pone en una situación no solo de violencia en contra de su integridad física y psíquica y afectación de sus derechos sexuales y reproductivos, sino también en una situación de riesgo para su vida.

Las diversas regulaciones apuntan a la visión tradicional que liga los DD. HH. a la existencia misma de los individuos y que, en consecuencia, afirma que aquellos se les deben reconocer y respetar a todos los individuos de la especie humana en todos los lugares del globo terrestre. Esta postura se refleja claramente en la Declaración Universal de los Derechos Humanos, de cuyo nombre se deduce fácilmente su pretensión de universalidad. Dichas pretensiones bien pueden ser revisadas en sus raíces, desde la imposibilidad de determinar con grado de certeza la base, el contenido o los alcances de determinados derechos (Vargas y Bracchi, 2016). Afirmamos que esa pretendida universalidad encuentra su sustento en la filosofía, más que en la práctica, el desarrollo y la garantía de estos derechos.

Ese rasgo elemental al que apunta la universalidad, y que se circunscribe en la esfera de lo filosófico, tiene como consecuencia el que los derechos humanos no sean concebidos de forma estricta como derechos jurídicos, sino más bien, y sobre todo, como derechos morales, es decir, como derechos a los que corresponde una determinada obligación y una determinada sanción —en caso de que se incumpla con la obligación—, pero que no gozan necesariamente del apoyo por parte del aparato institucional del Estado. ${ }^{19}$ (Dávila, 2013, p. 36)

18 Traducción libre del original en inglés: Female genital mutilation deprives girls and women from making an independent decision about an intervention that has a lasting effect on their bodies and infringes on their autonomy and control over their lives.

19 Acerca de los modelos de fundamentación absoluta en materia de universalidad de los derechos humanos, el autor refiere dos modelos principales, el religioso y el contractualista, que parecen ser los más aceptados como justificación filosófica de la universalidad de los derechos humanos. 
A nuestro juicio, es posible reconducir la fundamentación filosófica de la universalidad de los derechos humanos, en la dignidad, que se encuentra en el germen de aquellos. Frente a las críticas respecto de la imposibilidad de llenar de contenido la dignidad, seguimos a Habermas cuando afirma que la dignidad es un concepto normativo, fundamental y sustancial.

La dignidad humana no es únicamente una expresión clasificatoria, como si se tratara de un parámetro de sustitución vacío que agrupara una multiplicidad de fenómenos diferentes. Por el contrario, [...] constituye la "fuente" moral de la que todos los derechos fundamentales derivan su sustento. (Habermas, 2010, p. 6)

No puede perderse de vista que el surgimiento de los DD. HH. estuvo fundamentado en las atrocidades derivadas de la segunda guerra mundial, y es allí donde el concepto de la dignidad humana — como señala Habermas - permitió acordar consensos en contextos culturales diversos que, de otra forma, no habría propiciado el surgimiento de declaraciones universales. Igualmente, en relación con los retos derivados de las nuevas realidades relacionadas con minorías culturales, religiosas, procesos migratorios, entre otros, que suponen importantes retos en materia de DD. HH., la dignidad ha fungido como material y sustento creativo.

La dignidad humana, lejos de presentarse como un concepto vacío de contenido, se ha transformado en un regulador de la validación práctica de los derechos, con un papel protagónico (Maldonado, 2017). Al respecto, señala Habermas que

[...] la dignidad humana configura el portal a través del cual el sustrato igualitario y universalista de la moral se traslada al ámbito del derecho. La idea de la dignidad humana es el eje conceptual que conecta la moral del respeto igualitario de toda persona con el derecho positivo y el proceso de legislación democrático, de tal forma que su interacción puede dar origen a un orden político fundado en los derechos humanos. (Habermas, 2010, p. 10)

La dignidad humana es, entonces, el principio iluminador que permite, de un lado, reafirmar la universalidad de los derechos humanos y, de otro lado, salirles al paso a las 
críticas en relación con los contornos difusos que el catálogo de DD. HH. pareciera tener, en su aplicación en contextos culturales, sociales y religiosos diversos. En ese sentido, Nussbaum enfatiza que

[...] necesitamos también preguntar si el marco que proponemos, si un único universal, es suficientemente flexible para permitirnos hacer justicia para la variedad humana que encontramos. El reto es serio porque el desarrollo de proyectos internacionales a menudo ha fallado debido a la falta de sintonía con la variedad y particularidad cultural. ${ }^{20}$ (Nussbaum, 2000, p. 40)

Consideramos que el debate en torno a la MGF se mueve no desde la superioridad de culturas (indicando una distinción entre culturas superiores o inferiores), sino desde la universalidad de la categoría derechos humanos, en tanto pertenecientes a todos los seres humanos en relación con los estándares de dignidad que la humanidad ha definido como indispensables (Valderrama, 2016). Insistimos en que debe cuidarse la utilización del discurso de los derechos humanos con un enfoque de superioridad, o con una interpretación desde la supuesta supremacía de ciertas culturas sobre otras (Garzón, 2013).

La aplicación de los derechos humanos debería centrarse en preservar la dignidad humana. Para ello, se ha de realizar un minucioso trabajo etnográfico sobre el significado social, simbólico y económico de la MGF, en lugar de demonizar a quienes la practican. Dicha estrategia constituye una oportunidad para construir un diálogo intercultural introspectivo y profundo que respete la dignidad de las personas (Santinho, 2017, p. 103).

\section{CONCLUSIONES}

La MGF es una práctica que, en sus diversas modalidades, implica graves consecuencias para la salud física, sexual, obstétrica y reproductiva de mujeres y niñas; además, puede

20 Traducción libre del original en inglés: And we also need to ask whether the framework we propose, if a single universal one, is sufficiently flexible to enable us to do justice to the human variety we find. This challenge is serious because international development projects have often gone wrong through insufficient attunement to cultural variety and particularity. 
producir afectaciones psicológicas. Las condiciones en las que se practica rayan con elementos que pueden catalogarse como tortura de nińas y mujeres.

La MGF representa la concreción de una sociedad que discrimina y violenta a las mujeres, y la dificultad principal para erradicarla se encuentra en que se trata de una práctica tradicional, ligada a la cultura de los grupos humanos.

La MGF constituye una violación de los DD. HH., en especial de los derechos a la vida, a la salud, a una vida libre de violencias y de discriminación; los organismos internacionales han exhortado a los Estados para que formulen políticas y planes encaminados a su erradicación.

Pese a lo anterior, las políticas meramente legislativas y punitivas no son suficientes, sino que deben estar acompañadas de intervenciones sociales respetuosas de la cultura, la diversidad y las cosmovisiones de los grupos humanos que practican la MGF, ya que se trata de una práctica acendrada en la cultura. Por esta razón, el proceso de eliminación debe centrarse en la priorización de la salud de las mujeres y niñas, sin apelar a discursos etnocentristas o de superioridad cultural.

\section{REFERENCIAS}

Althaus, F. A. (1997). Female circumcision: rite of passage or violation of rights? International Perspectives on Sexual and Reproductive Health, 23(3), 130. Recuperado de from https://www.guttmacher.org/journals/ipsrh/1997/09/female-circumcision-rite-passageor-violation-rights

Amnistía Internacional. (1997). La mutilación genital femenina y los derechos humanos: infibulación, excisión y otras prácticas cruentas de iniciación. Recuperado de https:// www.corteidh.or.cr/tablas/12056.pdf

Asociación Mujeres entre Mundos. (2016). Guía para la concienciaión sobre mutilaciones genitales femeninas. Recuperado de https://www.juntadeandalucia.es/export/drupaljda/ Guia_Mutilacion_Genital_Femenina_0.pdf 
Ballesteros, A. (2015). La mujer y el ejercicio efectivo del poder en Colombia: una cuestión de derechos humanos. Verba Iuris, (33). Recuperado de https://revistas.unilibre.edu.co/ index.php/verbaiuris/article/view/27/24

Behrendt, A. y Moritz, S. (2005). Posttraumatic stress disorder and memory problems after female genital mutilation. American Journal of Psychiatry, (162), 1000-1002. Recuperado de https://www.taskforcefgm.de/wp-content/uploads/2010/03/FGM_Trauma.pdf

Bennett, S. (n. d.). Female genital mutilation/cutting. Recuperado de http://childrenshealthcare.org/wp-content/uploads/2010/11/Susan-Bennett-Chapter-17-11-15-10.pdf

Boyle, E. H. (2005). Female genital cutting: cultural conflict in the global community. Baltimore, MD: Johns Hopkins University Press. Recuperado de https://ebookcentral-proquestcom.ezproxy.umng.edu.co/lib/umng-ebooks/detail.action?docID=3318171

Dávila, J. A. (2013). La universalidad de los derechos humanos y su fundamentación absoluta: una visión crítica. Analecta Política, 3(4), 33-54.

Dorkenoo, E. (1996). Combating female genital mutilation: an agenda for the next decade. World Health Statistics Quarterly. Rapport Trimestriel de Statistiques Sanitaires Mondiales, 49(2), 142-147.

Garzón, E. (2013). Globalización del derecho, fetichismo legal el velo de los derechos humanos. Verba Iuris, (30), 56-78.

Glover, J., Liebling, H., Barrett, H. y Goodman, S. (2017). The psychological and social impact of female genital mutilation: a holistic conceptual framework. Journal of International Studies, 10(2), 219-238. https://doi.org/https://doi. org/10.14254/2071-8330.2017/10-2/16

Gomez-Limón, M. T. (2011). Las tradiciones que no aman a las mujeres. Madrid: Akal.

Habermas, J. (2010). El concepto de dignidad humana y la utopía realista de los derechos humanos. Diánoia, 55(64), 3-25.

Hakin, L. Y. (2001). Impact of female genital mutilation on maternal and neonatal outcomes during parturition. East African Medical Journal, 78(5), 255-258. https://doi.org/ https://doi.org/10.4314/eamj.v78i5.9049 
Jones, H., Diop, N., Askew, I. y Kaboré, I. (2003). Female genital cutting practices in Burkina Faso and Mali and their negative health outcomes. Studies in Family Planning, 30(3), 219-230. https://doi.org/https://doi.org/10.1111/j.1728-4465.1999.00219.x

Kaplan, A., Nuño Gómez, L., Aliaga, N., Bermejo Casado, R., Carvalho, C., Cavatorta, G., ... Kaplan, A. (2017). Guí multisectorial de formación académica sobre mutilación genital femenina (M. Thill y N. S. Seoane, Eds.). Madrid: Dykinson. Recuperado de https:// mapfgm.eu/wp-content/uploads/2017/04/Guia-Castellano.pdf

La Barbera, M. C. (2010). Intervenciones sobre los genitales femeninos. Revista de Dialectologia y Tradiciones Populares, 75(2), 465-488. Recuperado de https://dialnet.unirioja.es/ servlet/articulo? codigo $=3357754$

Maldonado, G. (2017). La afiliación fraudulenta en el sistema de seguridad social integral colombiano. Via Inveniendi et Iudicandi, 12(2), 73-99. https://doi.org/https://doi. org/10.15332/s1909-0528.2017.0002.04

Nussbaum, M. C. (2000). Women and human development. The capabilities approach. Cambridge: Cambridge University Press. Recuperado de https://genderbudgeting.files.wordpress.com/2012/12/nussbaum_women_capabilityapproach2000.pdf

Organización Mundial de la Salud (oms). (2006). Female genital mutilation and obstetric outcome: WHO collaborative prospective study in six African countries. The Lancet, (367), 1835-1841. Recuperado de https://www.who.int/reproductivehealth/publications/fgm/fgm-obstetric-study-en.pdf

Organización Mundial de la Salud (OMs). (2008). Eliminating female genital mutilation, an interagency statement. Ginebra: OMs. Recuperado de https://apps.who.int/iris/bitstream/ handle/10665/43839/9789241596442_eng.pdf;jsessionid=66648168539D8AD8FD4 7ED43FE68E9D8? sequence $=1$

Organización Mundial de la Salud (oms). (2020). Mutilación genital femenina. Recuperado de https://www.who.int/es/news-room/fact-sheets/detail/female-genital-mutilation

Paakkanen, E. K. (2019). Entitled, empowered or victims - an analysis of discourses on male and female circumcision, genital mutilation/cutting and genital cosmetic surgery. The International Journal of Human Rights, 23(9), 1494-1520. https://doi.org/10.1080/136 42987.2019.1612375 
Santinho, C. (2017). Multiculturalismo y derechos humanos. En Guía multisectorial de formación académica sobre mutilación genital femenina (pp. 103-104). Recuperado de mapfgm.eu/wp-content/uploads/2017/04/Guia-Castellano.pdf

Unfra. (2019). Preguntas frecuentes sobre la mutilación genital femenina. Recuperado de https://www.unfpa.org/es/resources/preguntas-frecuentes-sobre-la-mutilacióngenital-femenina-mgf\#Qué-consecuencias

Unicef. (2005). Cambiar una convención social perjudicial: la ablación o mutilación genital femenina (C. de I. I. Unicef, Ed.). Unicef. Recuperado de https://www.unicef-irc.org/ publications/pdf/fgm-e.pdf

Utz-Billing, I. y Kentenich, H. (2008). Female genital mutilation: an injury, physical and mental harm. Journal of Psychosomatic Obstetrics and Ginecology, 29(4), 225-229. https:// doi.org/10.1080/01674820802547087

Valderrama, I. (2016). El principio de congruencia en el proceso penal. Via Inveniendi et Iudicandi, 11(2), 159-180.

Valderrama, I., Téllez, R. y Blanco, C. (2018). La incertidumbre de las victimas dentro del acuerdo para la construcción de una paz estable y duradera. En Tendencias actuales de los derechos humanos y el derecho internacional humanitario en Colombia (pp. 83103). Recuperado de https://repository.usta.edu.co/bitstream/handle/11634/13049/ Obracompleta.2018Carvajaljorge.pdf?sequence $=1$ \&isAllowed $=y$

Vargas, Y. y Bracchi, C. (2016). La mujer latinoamericana dentro del contexto de los derechos humanos: énfasis desde la perspectiva del derecho al trabajo. IUSTA, 1(44), 75-97.

Yoder, P. S., Abderrahim, N. y Zhuzhuni, A. (2004). Female genital cutting in the demographic and health surveys: a critical and comparative analysis. DHS Comparative Reports No. 7. Recuperado de https://dhsprogram.com/pubs/pdf/CR7/CR7.pdf 